\title{
Estructuras de titanio con gradiente de porosidad - fabricación por el método de partículas espaciadoras y caracterización
}

\section{Titanium structures with porosity gradient - space holder manufacturing and characterization}

\author{
${ }^{1}$ Departamento de Ingeniería Metalúrgica, Facultad Córdoba, Universidad Tecnológica Nacional, Córdoba, Argentina. \\ e-mail: robertolucci1984@gmail.com \\ 2 Departamento de Materiales y Tecnología, FCEFN, Universidad Nacional de Córdoba, Córdoba, Argentina. \\ e-mail: coldani@efn.uncor.edu
}

\section{RESUMEN}

Se ha propuesto el uso de titanio poroso para reducir el problema de incompatibilidad mecánica que ocurre entre el implante y el hueso. La introducción de poros en el titanio disminuye su módulo elástico acercándolo al que poseen tanto los huesos corticales como trabeculares.

En este trabajo se investigó la fabricación por pulvimetalurgia de titanio con gradiente de porosidad longitudinal, usando carbonato ácido de amonio como material espaciador, mezclado en distintas proporciones con hidruro de titanio en polvo. Luego de la compactación uniaxial por capas y posterior sinterización a $1150{ }^{\circ} \mathrm{C}$, se obtuvieron muestras cilíndricas con porosidad longitudinal gradual de proporciones $2 \%-30 \%, 2 \%$ $60 \%, 30 \%-60 \%$ y $2 \%-30 \%$ - $60 \%$. Se analizó la microestructura; tamaño, forma y distribución de poros, así como el módulo de elasticidad y límite de fluencia.

Los resultados indican que, mediante el método estudiado, es posible obtener muestras sinterizadas con porosidad gradual, con continuidad estructural entre las distintas capas porosas, con un rango de tamaño de poro entre 200 y $250 \mu \mathrm{m}$. Los valores de módulo de elasticidad obtenidos fueron similares a los informados en la bibliografía para los huesos corticales y son compatibles con la estructura anisotrópica del tejido óseo.

Palabras clave: Biomateriales, titanio, gradiente de porosidad, sinterización

\begin{abstract}
It has proposed the use of porous titanium to reduce the problem of mechanical incompatibility, which occurs between such material used in implants and bone. The pores introduction in titanium decreases its elastic modulus by bringing it closer to that of both cortical and trabecular bones.

This paper describes titanium fabrication by powder metallurgy with longitudinal porosity gradient, using as space holder material, ammonium hydrogen carbonate, in different proportions, mixed with titanium hydride powder. After layered uniaxial compaction and subsequent sintering at $1150{ }^{\circ} \mathrm{C}$, cylinders with gradual longitudinal porosity $2 \%-30 \%, 2 \%-60 \%, 30 \%-60 \%$ and $2 \%-30 \%-60 \%$ were obtained. Microstructure, size, shape and pore distribution, elasticity modulus and yield stress were analyzed in the samples.

The results indicate that by the method studied, it is possible to obtain sinterized samples with porosity gradient, structural continuity between the different porous layers, with a range of pore size between 200 and 250 $\mu \mathrm{m}$. The elasticity modulus in samples was similar to those reported in the literature for cortical bones and is compatible with the bone tissue anisotropic structure.
\end{abstract}

Keywords: Biomaterials, titanium, porosity gradient, sintering. 
Muchas fallas de los implantes de titanio están asociadas con el fenómeno de retracción ósea o "stress shielding", debido a la diferencia de rigidez entre el hueso y el implante [1-2]. La posible disminución de este fenómeno ha sido tratada a través de dos caminos [3]: se pueden encontrar trabajos de investigación que están enfocados en el desarrollo de nuevos materiales para implantes con bajo módulo elástico, acercándose al del hueso, basados en aleaciones de titanio- $\beta$ metaestables [4-5]. Otra línea de trabajo es la de disminuir la rigidez del Ti mediante la incorporación de poros al material [6-10]. Dentro de los diversos métodos desarrollados para la formación de poros, uno de los más usados por su simpleza es el de partículas espaciadoras [7, 11-12].

En este escenario, la pulvimetalurgia da la posibilidad de conseguir un material con porosidad regulada en forma de gradiente. El diseño de nuevas generaciones de titanio con gradiente de porosidad longitudinal como radial, es una dirección de trabajo promisoria para conseguir un balance de propiedades mecánicas que asegure una solución al tema del stress shielding [7,13]. Adicionalmente, la alta porosidad superficial facilita el ingreso directo del hueso [14-15], la infiltración con diferentes sustancias que van a ayudar a conseguir un balance entre la osteointegración, el stress shielding y las propiedades mecánicas del implante.

El objetivo de este trabajo fue desarrollar estructuras porosas de titanio con gradiente de porosidad utilizando el método de partículas espaciadoras, su caracterización por microscopía óptica y electrónica y su comportamiento mecánico mediante ensayos de compresión.

El método pulvimetalúrgico estudiado consistió en la compactación de capas de, mezclas de hidruro de titanio mezclado con carbonato ácido de amonio en polvo en distintas proporciones, para obtener compactos "en verde", que posteriormente fueron sinterizados para lograr estructuras con gradiente de porosidad.

\section{MATERIALES Y MÉTODOS}

Se utilizó hidruro de titanio en polvo (proveedor Sigma Aldrich) con una pureza del $98 \%$, con tamaño de partícula menor a $43 \mu \mathrm{m}$. Como material espaciador se usó carbonato ácido de amonio puro en polvo (proveedor Anedra), con tamaño de partícula tamizada con malla de apertura $250 \mu \mathrm{m}$. La mezcla de los polvos de $\mathrm{TiH}_{2}$ y $\mathrm{NH}_{4} \mathrm{HCO}_{3}$ se llevó a cabo en un mezclador de vidrio tipo Y. Se utilizaron diferentes proporciones de carbonato ácido de amonio - hidruro de titanio, para obtener distintos valores de porosidad en cada una de las capas de la muestra a preparar. Las masas de $\mathrm{TiH}_{2}$ y $\mathrm{NH}_{4} \mathrm{HCO}_{3}$ utilizadas para obtener las porosidades de 2 $\%, 30 \%$ y $60 \%$ se indican en la Tabla 1.

Tabla 1: Masas de $\mathrm{TiH}_{2}$ y $\mathrm{NH}_{4} \mathrm{HCO}_{3}$ requeridas en la mezcla de los polvos, para obtener muestras con porosidades variables de $2 \%, 30 \%$ y $60 \%$.

\begin{tabular}{l|l|l}
\hline $\begin{array}{l}\text { POROSIDAD } \\
(\%)\end{array}$ & $\begin{array}{l}\text { MASA } \mathrm{TiH}_{2} \\
(\mathbf{g})\end{array}$ & $\begin{array}{l}\text { MASA } \mathrm{NH}_{4} \mathrm{HCO}_{3} \\
(\mathbf{g})\end{array}$ \\
\hline 2 & 1,18 & 0 \\
\hline 30 & 0,82 & 0,15 \\
\hline 60 & 0.47 & 0,30 \\
\hline
\end{tabular}

Con posterioridad al mezclado, se vaciaron dentro de una matriz de acero cilíndrica de $8 \mathrm{~mm}$ de diámetro, capas de igual espesor (alrededor de $3 \mathrm{~mm}$ cada una) de las mezclas con distintas relaciones $\mathrm{TiH} 2$ y NH4HCO3, para obtener los gradientes de porosidad buscados. La compactación uniaxial de los polvos cargados en la matriz fue realizada a una presión de $500 \mathrm{MPa}$. Los compactos en verde obtenidos en la compactación uniaxial fueron llevados a un horno tubular de calentamiento eléctrico marca Sentro Tech, com una bomba de vacío mecánica rotativa de doble etapa Edwards RV 3 y una bomba difusora de aceite Edwards Diffstak 63/150. Los compactos se sometieron a calentamiento a una velocidad de $7{ }^{\circ} \mathrm{C}$ por minuto, en vacío de 10-5 mm de $\mathrm{Hg}$. La primera etapa de calentamiento se realizó a $200{ }^{\circ} \mathrm{C}$ durante una hora para eliminar el bicarbonato de amonio por descomposición de acuerdo a la reacción (1), dejando un compacto de hidruro de titanio poroso con poros interconectados.

$\mathrm{NH} 4 \mathrm{HCO} 3=\mathrm{NH} 3 \uparrow+\mathrm{CO} 2 \uparrow+\mathrm{H} 2 \mathrm{O} \uparrow$

La segunda etapa, consistió en mantener la muestra una hora a $600{ }^{\circ} \mathrm{C}$ para descomponer el hidruro de titanio y transformarlo en titanio metálico eliminando el hidrógeno. La última etapa fue realizada a $1150{ }^{\circ} \mathrm{C}$ 
durante dos horas para producir la sinterización de los polvos de titanio y obtener las propiedades mecánicas buscadas.

Las muestras sinterizadas se estudiaron metalográficamente mediante microscopía confocal láser. Posteriormente, se efectuaron ensayos mecánicos de compresión y análisis de superficie de fractura de las muestras ensayadas mecánicamente. La evaluación del módulo de elasticidad y límite de fluencia de las muestras con gradiente de porosidad fue realizada a partir de ensayos de compresión. Se determinaron estas propiedades aplicando la carga sobre los compactos cilíndricos de dimensiones de $7 \mathrm{~mm}$ de diámetro por $7 \mathrm{~mm}$ de altura, tanto en sentido longitudinal como transversal al gradiente de porosidad. Los resultados obtenidos en los ensayos corresponden al valor promedio de 3 muestras utilizadas en cada determinación. El equipo usado en la realización de los ensayos de compresión fue una máquina de ensayos universal Instron modelo 4486. Los ensayos fueron realizados utilizando una velocidad de deformación nominal de 10-2 s-1.

\section{RESULTADOS}

\subsection{METALOGRAFÍA}

En la Figura 1 se muestra una micrografía correspondiente al corte transversal de una muestra cilíndrica de titanio sinterizado con gradiente de porosidad, en la cual se distinguen tres capas bien diferenciadas. Se observa que la capa de la derecha no posee partículas separadoras de $\mathrm{NH}_{4} \mathrm{HCO}_{3}$ y que gradualmente la porosidad (P) va aumentando hacia la izquierda. Se puede apreciar que la muestra es sana, compuesta por una matriz de titanio alfa (Ti $\alpha$ ), con continuidad estructural entre las distintas capas porosas, sin presencia de fisuras o segregaciones en las interfaces. Mediante el uso del analizador de imágenes Image Pro Plus 6.1, se determinó un rango de tamaño de poros entre 200 y $250 \mu \mathrm{m}$.



Figura 1: Micrografía tomada con microscopio confocal láser en la muestra de titanio sinterizado con gradiente de porosidad $2 \%-30 \%-60 \%$.

En la Figura 2 se presentan sectores de la muestra de la Figura 1 con distinta porosidad. En el sector (a), se observa una estructura muy porosa de poros interconectados $(60 \%)$. En el sector (b), con una porosidad del $30 \%$, se observa un importante porcentaje de fase metálica. Finalmente, en el sector (c) correspondiente a la sinterización de titanio sin partículas separadoras, se observa una estructura muy compacta, con baja porosidad $(2 \%)$ y sin poros de gran tamaño. 


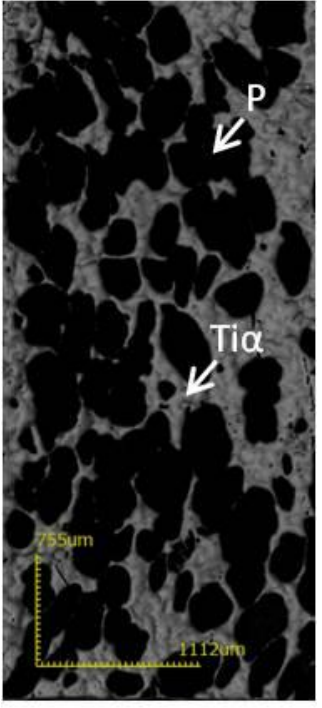

(a)

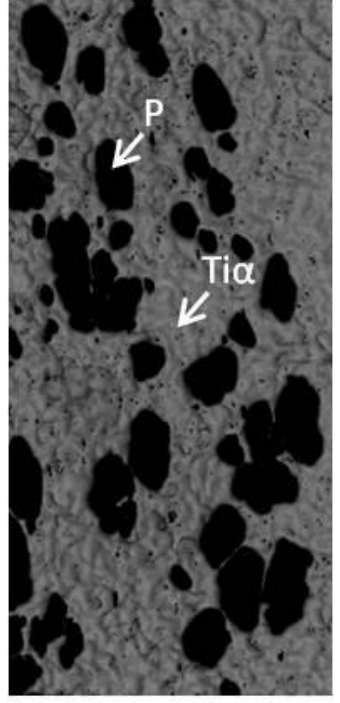

(b)

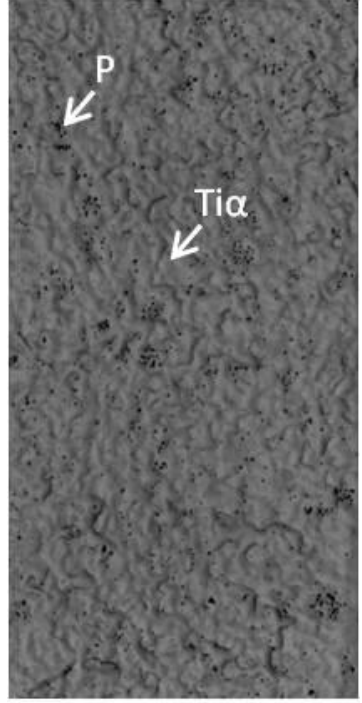

(c)

Figura 2: Secciones de la muestra con distinto grado de porosidad: (a) $60 \%$, (b) $30 \%$, (c) $2 \%$.

En la Figura 3 se muestran micrografías del interior de una muestra fracturada, obtenidas por microscopía electrónica. En las mismas se observa una estructura de poros $(\mathrm{P})$ altamente rugosa y cavernosa, adecuada para la oseointegración. También se observan microporos de menos de $10 \mu \mathrm{m}$ de diámetro producidos durante la sinterización incompleta de las partículas de titanio.

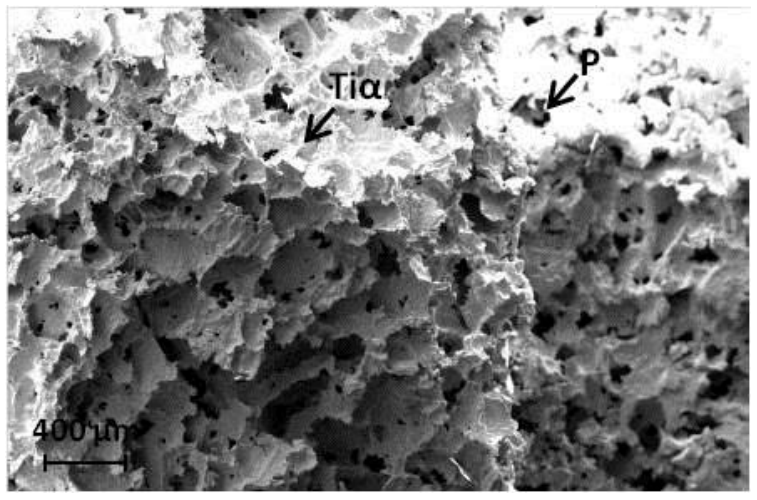

(a)



(b)

Figura 3: Superficie interna de compacto de titanio de $60 \%$ de porosidad.

\subsection{PROPIEDADES MECÁNICAS}

En la Tabla 2 se muestran los valores obtenidos en los ensayos de compresión.

Tabla 2: Módulos de elasticidad y límites de fluencia obtenidos en compactos con gradientes de porosidad.

\begin{tabular}{l|l|l}
\hline GRADIENTE DE POROSIDAD (\%) & MÓDULO DE ELASTICIDAD (GPa) & TENSIÓN DE FLUENCIA (MPa) \\
\hline $2-30-60$ (Carga longitudinal) & 20 & 200 \\
\hline $2-30-60$ (Carga transversal) & 43 & 500 \\
\hline $30-60$ (Carga transversal) & 21 & 150 \\
\hline $2-30$ (Carga transversal) & 46 & 550 \\
\hline
\end{tabular}

A modo de comparación con los valores de la Tabla 2, se muestran en la Tabla 3, los valores de módulo de elasticidad y tensión de fluencia obtenidos en compactos porosos de titanio con porosidad uniforme 
informados en trabajo anterior [3].

Tabla 3: Módulos de elasticidad y límites de fluencia obtenidos en compactos con porosidad uniforme.

\begin{tabular}{l|l|l}
\hline POROSIDAD (\%) & MÓDULO DE ELASTICIDAD (GPa) & TENSIÓN DE FLUENCIA (MPa) \\
\hline 2 & 98,5 & 600 \\
\hline 35 & 13,2 & 225 \\
\hline 50 & 6,8 & 110 \\
\hline 61 & 2,4 & 45 \\
\hline
\end{tabular}

Se puede apreciar que el módulo de elasticidad y tensión de fluencia disminuyen marcadamente en la muestra con gradiente de porosidad ensayada longitudinalmente respecto de los valores con $2 \%$ de porosidad uniforme (Tabla 3). Sin embargo, en las muestras ensayadas transversalmente al gradiente de porosidad, se nota que los valores del módulo de elasticidad y tensión de fluencia son superiores a los obtenidos en las muestras con porosidad uniforme.

\section{DISCUSIÓN}

La estructura alcanzada en las muestras porosas de titanio obtenidas a través del proceso estudiado, imita la arquitectura que se observa en el tejido óseo, en el cual, en la parte exterior, se encuentra el hueso cortical que presenta baja porosidad y en el interior, el hueso trabecular mucho más poroso. También se observa en las muestras una microestructura porosa y rugosa con tamaño de poro entre 200 y $250 \mu \mathrm{m}$ y presencia de microporos. Según Taniguchi et al. [15], el rango de tamaño de poros alcanzado en los compactos obtenidos permite una buena migración celular, necesaria para el acceso de sangre, oxígeno, minerales, iones, hormonas, factores de crecimiento y nutrientes para la mineralización ósea y al mismo tiempo una buena adhesión celular requerida para la proliferación, migración y diferenciación del tejido óseo dentro del sustrato poroso.

Respecto a las propiedades mecánicas obtenidas, se observa que durante el ensayo de compresión en el sentido longitudinal de la muestra con gradiente de porosidad $2 \%$ - $30 \%$ - $60 \%$, tanto el módulo de elasticidad como el límite de fluencia de los compactos disminuye bastante respecto del valor del módulo de un material con muy baja porosidad y los mismos se encuentran dentro de los valores estudiados para los huesos corticales. Esta disminución en las propiedades mecánicas puede atribuirse a que las capas más porosas presentes en la muestra, ceden rápidamente bajo el efecto de la carga y recién cuando éstas se han comprimido bastante, comienza a actuar la carga sobre la zona de menor porosidad.

Al ensayar otra muestra con igual porosidad $2 \%-30 \%-60 \%$ pero en sentido transversal al gradiente se observó que los valores de módulo y límite de fluencia (43 GPa y $500 \mathrm{MPa}$ ), son superiores a los determinados longitudinalmente. Esto indicaría que la carga se distribuye más uniformemente sobre las tres capas de diferente porosidad.

Al ensayar en sentido transversal al gradiente de porosidad las muestras con dos capas de distinta porosidad, $2 \%$ - $30 \%$ y $30 \%$ - $60 \%$, se observa que la que presenta menor porosidad (2\% - 30\%), da valores superiores a las de mayor porosidad (30\%- $60 \%$ ). Este comportamiento tendría una explicación lógica al considerar que las muestras con capas más compactas ofrecen mayor resistencia a la deformación.

\section{CONCLUSIONES}

Mediante el método de sinterización estudiado fue factible producir titanio con gradiente de porosidad.

Se obtuvo una microestructura libre de discontinuidades o fisuras en las interfaces de las capas con distinta porosidad. Asimismo, se encontraron poros rugosos de alrededor de $200-250 \mu \mathrm{m}$ de diámetro que permitirían una adecuada oseointegración y vascularización para el crecimiento interno del hueso.

Se determinó que los valores de propiedades mecánicas obtenidas dependen de la dirección del ensayo respecto del gradiente de porosidad. Los valores de módulo elástico y tensión de fluencia en sentido transversal fueron similares, pero son superiores a los que poseen los huesos corticales. Sin embargo, para gradientes de $30 \%-60 \%$ de porosidad, se obtuvo un módulo de elasticidad dentro de los valores indicados para los huesos corticales. Los valores de de módulo elástico y tensión de fluencia longitudinal para gradientes de $2 \%$ - $30 \%$ - $60 \%$ se encuentran dentro de los recomendados para los huesos corticales (10-30 GPa y 200-250 $\mathrm{MPa})$. 


\section{AGRADECIMIENTOS}

Departamento de Ingeniería Metalúrgica, UTN-FRC, Córdoba, Argentina. Departamento de Materiales y Tecnología, FCEFN-UNC, Córdoba, Argentina.

\section{BIBLIOGRAFÍA}

[1] BRUNETTE, D. M., TENGVALI, P., TEXTOR, M., et al., Titanium in medicine: materials science, surface science, engineering, biological responses and medical applications, Berlin, Springer, 2001.

[2] TORRES, Y., TRUEBA, P., PAVÓN, J., et al., "Designing, processing and characterisation of titanium cylinders with graded porosity: An alternative to stress-shielding solutions", Materials and Design, v.63, pp. 316-324, 2014.

[3] OLDANI, C., DOMINGUEZ, A (2012). Titanium as a Biomaterial for Implants, Recent Advances in Arthroplasty, InTech, Available from: http://www.intechopen.com/books/recent-advances-inarthroplasty/titanium-as-a-biomaterial-for-implants, pp. 149-162, 2012.

[4] QAZI, J., RACK, H., MARQUARDT, B., "High-strength metastable beta-titanium alloys fort biomedical applications", JOM 56, v.56, n.11, pp. 49-51, 2004.

[5] GUILEMOT, F., PRIMA, F., LATTA, L., et al., "Design of new titanium alloys for orthopedic applications", Medical and Biological Engineering and Computing 42, pp. 137-141, 2004.

[6] SINGH, R., LEE, P., DASHWOOD, R., et al., "Titanium foams for biomedical applications: a review", Mater. Technol. 25, pp. 127-136, 2010.

[7] TRUEBA,P., CHICARDI, E., RODRIGUEZ-ORTIZ, J., et al., "Design, Processing and Characterization of Materials with Controlled Radial Porosity for Biomedical and Nuclear Applications", Key Engineering Materials 704, pp. 325-333, 2016.

[8] TRUEBA, P., BASCÓN SUÁREZ, J., RODRIGUEZ-ORTIZ, Y., J., et al, Diseño y fabricación de un dispositivo para la obtención de cilindros de ti con porosidad dirigida, In: VI Congreso Nacional de Pulvimetalurgia y I Congreso Iberoamericano de Pulvimetalurgia, Ciudad Real, Castilla España, Junio de 2017.

[9] MARINESCU, C., SOFRONIA, A., ANGHEL, E., et al., "Microstructure, stability and biocompatibility of hydroxyapatite - titania nanocomposites formed by two step sintering process", Arabian Journal of Chemistry, Available online 9/2/2017, In Press, Corrected Proof, open access.

[10] SOBIESZCZYK, S. "Optimal features of porosity of Ti alloys considering their bioactivity and mechanical properties", Advance Materials Science, v. 10, pp. 100-110, 2010.

[11] JHA, N., et al., "High porous open cell Ti-foams using $\mathrm{NaCl}$ as temporary space holder through powder metallurgy route", Mater. Des 47, pp. 810-819, 2013.

[12] LI, B., WANG, C., LU,X. "Effect of the pore structureon the compressive properties of porous Ti produced by powder mwtallurgy technique", Mater. Des., v.50, pp. 613-619, 2013.

[13] ARABNEJAD, S., JOHNSTON, R., PURA, J., et al., High-strength porous biomaterials for bone replacement: A strategy to assess the interplay between cell orphology, mechanical properties, bone ingrowth and manufacturing constraints, Acta Biomaterialia 30, pp. 345-356, 2016.

[14] OLDANI, C., et al., "Estudio de Ti, Ti poroso y composite Ti-HA: tests in-vitro e in-vivo", In: Congreso de la Sociedad Argentina de Bioingeniería, Córdoba, Argentina, oct., 2017.

[15] TANIGUCHI, N., FUJIBAYASHI, S., TAKEMOTO, M., et al., "Effect of pore size on bone ingrowth into porous titanium implants fabricated by additive manufacturing: An in vivo experiment", Materials Science and Engineering $C$, v. 59, pp. 690-701, 2016. 